\title{
Risk of hip, subtrochanteric, and femoral shaft fractures among mid and long term users of alendronate: nationwide cohort and nested case-control study
}

\author{
Bo Abrahamsen,,2 Pia Eiken,, 4 Daniel Prieto-Alhambra, 5, 6 Richard Eastell7
}

For numbered affiliations see end of article.

Correspondence to:

D Prieto-Alhambra, Botnar

Research Centre, NDORMS,

University of Oxford, Nuffield

Orthopaedics Centre, Oxford

OX3 7LD, UK

Daniel.prietoalhambra@

ndorms.ox.ac.uk

Cite this as: BMJ 2016;353:i3365

http://dx.doi.org/10.1136/bmj.i3365

Accepted: 16 May 2016

\section{ABSTRACT}

\section{OBJECTIVES}

To determine the skeletal safety and efficacy of long term ( $\geq 10$ years) alendronate use in patients with osteoporosis.

DESIGN

Open register based cohort study containing two nested case control studies.

SETTING

Nationwide study of population of Denmark.

\section{PARTICIPANTS}

61990 men and women aged 50-94 at the start of treatment, who had not previously taken alendronate, 1996-2007.

\section{INTERVENTIONS}

Treatment with alendronate.

\section{MAIN OUTCOME MEASURES}

Incident fracture of the subtrochanteric femur or femoral shaft (ST/FS) or the hip. Non-fracture controls from the cohort were matched to fracture cases by sex, year of birth, and year of initiation of alendronate treatment. Conditional logistic regression models were fitted to calculate odds ratios with and without adjustment for comorbidity and comedications. Sensitivity analyses investigated subsequent treatment with other drugs for osteoporosis.

RESULTS

1428 participants sustained a ST/FS (incidence rate $3.4 / 1000$ person years, $95 \%$ confidence interval 3.2 to 3.6), and 6784 sustained a hip fracture $(16.2 / 1000$ person years, 15.8 to 16.6$)$. The risk of ST/FS was lower

\section{WHAT IS ALREADY KNOWN ON THIS TOPIC}

Placebo controlled randomised clinical trials have shown a reduction in risk of hip fracture with alendronate treatment compared with placebo for three years in postmenopausal women with osteoporosis

Observational studies have suggested that atypical femur fractures involving the subtrochanteric femur or the femoral shaft are more common in long term users of bisphosphonates

\section{WHAT THIS STUDY ADDS}

Long term adherent use of alendronate in excess of 10 dose years was associated with an adjusted $30 \%$ lower risk of hip fracture and no increase in the risk of fractures of the subtrochanteric femur or femoral shaft

Even if all subtrochanteric and femoral shaft fractures observed in alendronate users were atypical, the number remains too low to offset the benefits on hip fracture with long term alendronate treatment for up to 10 years

The findings support a good benefit:risk with alendronate in terms of bone health for over 10 years of continuous use with high adherence to treatment with alendronate (medication possession ratio (MPR, a proxy for compliance) $>80 \%$ ) compared with poor adherence (MPR $<50 \%$; odds ratio $0.88,0.77$ to $0.99 ; \mathrm{P}=0.05$ ). Multivariable adjustment attenuated this association (adjusted odds ratio $0.88,0.77$ to $1.01 ; \mathrm{P}=0.08$ ). The risk was no higher in long term users ( $\geq 10$ dose years; $0.70,0.44$ to $1.11 ; P=0.13$ ) or in current compared with past users (0.91, 0.79 to $1.06 ; P=0.22)$. Similarly, MPR $>80 \%$ was associated with a decreased risk of hip fracture $(0.73,0.68$ to $0.78 ; \mathrm{P}<0.001)$ as was longer term cumulative use for $5-10$ dose years $(0.74,0.67$ to $0.83 ; \mathrm{P}<0.001)$ or $\geq 10$ dose years $(0.74,0.56$ to 0.97 ; $\mathrm{P}=0.03)$.

\section{CONCLUSIONS}

These findings support an acceptable balance between benefit and risk with treatment with alendronate in terms of fracture outcomes, even for over 10 years of continuous use.

\section{Introduction}

The clinical management of osteoporosis has progressed greatly in the past four decades with the introduction of potent antiresorptive drugs that substantially reduce the risk of fractures in men and women with osteoporosis. ${ }^{1}$ Despite the relatively low cost of such intervention, the treatment gap is widening in many areas of the world because of concerns that antiresorptive treatment could lead to atypical femur fractures $^{2}$ and that this could offset the benefits of long term use. ${ }^{3}$ Prescription rates for bisphoshonates have declined by $50 \%$ in the United States ${ }^{4}$ and similar trends have been observed in the European Union. ${ }^{5} \mathrm{~A}$ recent commentary in The BMJ concluded that bisphosphonates could achieve at best a marginal reduction in the risk of hip fracture and that the risk of serious medical adverse events, including atypical femur fractures, makes pharmacotherapy non-viable as a health strategy against hip fractures. ${ }^{6}$

Fractures classified as atypical femur fractures are defined by a set of clinical and radiological criteria, but they are also confined to a distinct anatomical area. Atypical femur fractures are substantially transverse fractures originating at the lateral femoral cortex. They are always non-comminuted and usually accompanied by localised or general cortical thickening. ${ }^{2}$ These fractures are a legitimate concern for prescribers and regulatory authorities given the short duration of the primary licensing trials for bisphosphonates. Trials typically lasted three years, with a limited proportion of participants carried forward into extension studies yielding a total follow-up length of 5-10 years, ${ }^{78}$ providing 
somewhat limited power to detect uncommon unexpected adverse events. ${ }^{9}$ The seemingly large number of atypical femur fractures predicted by odds ratios extracted from recent observational studies, ${ }^{10}$ however, seem to conflict with the observed slow increase and low overall rate of subtrochanteric and shaft fractures, the anatomical locations where atypical fractures would appear. ${ }^{11} 12$

To provide the best possible strategies for care and an optimum use of resources it is vitally important to recognise and verify safety alerts and, if verified, scale them appropriately so that clinical practice-including duration of osteoporosis treatment-is driven by absolute rates of benefit and absolute measures of harm so that treatments with good safety records are not inappropriately withdrawn or changed to less cost effective or less safe alternatives. Established in 1995, the Danish prescription registry uniquely holds almost 20 years of drug exposure data for all residents in the country, ${ }^{13}$ and this can be linked to all fracture outcomes treated in hospital in the same period. We used this unique real world data source to study the association between long term use of alendronate and the risk of subtrochanteric and femoral shaft fractures.

\section{Methods}

Study design

We used a nationwide population based open registry cohort study, containing two nested case-control studies, to determine the risk of subtrochanteric and femoral shaft and hip fractures as a function of cumulative alendronate use, time, and adherence to assess long term skeletal safety and efficacy.

\section{Study population and follow-up}

The included population was 63774 treatment naive incident users of alendronate in 1996-2007 in Denmark who were aged 50-94 at the time of start treatment (fig 1). Previous use of alendronate or other osteoporosis drugs was an exclusion criterion and drug exposures were tracked back to the establishment of the prescription registry in January 1995 . We excluded patients who moved away from Denmark $(n=1773)$ in the observation period (1996-2013) and those who were registered with impossible information such as a posthumous first prescription ( $\mathrm{n}=11)$, leaving a study population of 61990 patients exposed to alendronate. Patients in the cohort were followed from the start of treatment (that is, first prescription) until the earliest of death, transfer out, or end of study (31 December 2013). As the databases are event based and capture hospital contacts and filled prescriptions, there were no identifiably missing data.

Two case-control studies were nested within the cohort of alendronate users. Firstly, all patients who sustained a hip fracture in the study period were matched to up to three controls by age, sex, year of start of treatment, and follow-up time. Similarly, those who experienced a subtrochanteric/shaft fracture were identified as cases in a second case-control dataset and matched to up to five controls by the same four variables.

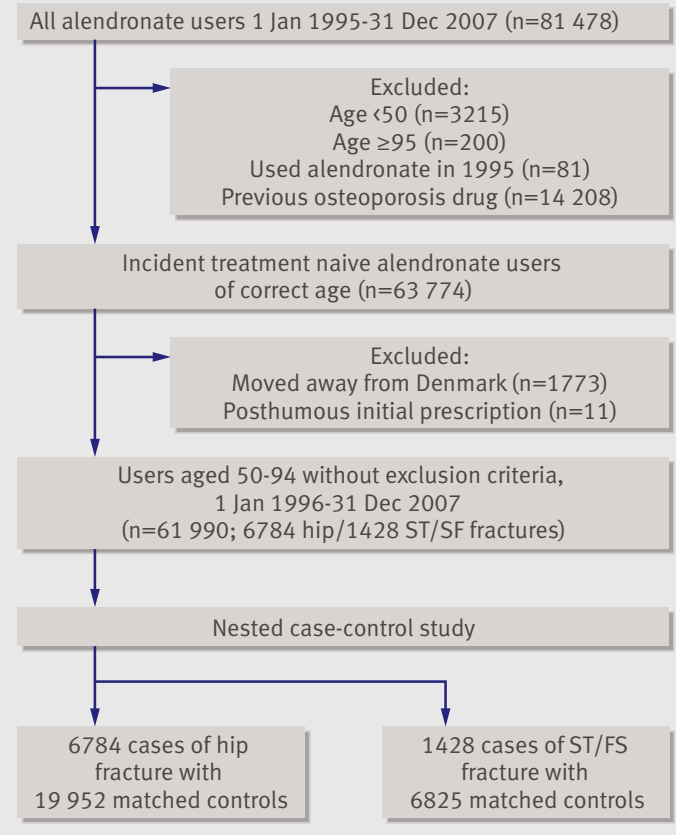

Fig 1 | Flow of patients using alendronate in study to determine effect on fractures

\section{Outcomes}

Outcomes were incident fractures of the hip (ICD-10 (international classification of diseases, 10th revision) codes S720 and S721), subtrochanteric femur (S722), and the femoral shaft (S723) recorded in the Danish Hospital Discharge Register maintained by the Danish National Board of Health. Incident outcomes were included in the analysis irrespective of current treatment status so that we could determine the association with strength of exposure (dose years, present user or not, medication possession ratio (MPR) a proxy for compliance).

\section{Exposures}

The key exposure was pharmacy dispensations for alendronate (ATC (Anatomical Therapeutic Chemical) codes M05BA04 and M05BB03) filled in 1996-2013 as assessed through the National Prescription Database. The register contains information about the dose and number of tablets for all drugs dispensed irrespective of whether the prescription originated in hospitals, private clinics, or from primary care physicians. The medication possession ratio was calculated as the number of WHO defined daily doses divided by the length of time in days for each year of treatment, transferring any excess doses (>365 defined daily doses in a year) into the next year, where it was added to prescriptions filled. The dose of alendronate used in Denmark is always $70 \mathrm{mg}$ a week-that is, a defined daily dose of $10 \mathrm{mg}$-as the $5 \mathrm{mg}$ daily preventive dose for osteopenia is not licensed there. Hence, for calculations of cumulative alendronate use, we converted the total number of defined daily doses filled to dose years by dividing by 365 . 


\section{Confounders}

Comedications considered for multivariable adjustment in the case-control studies included prednisolone, prednisone, and proton pump inhibitors. Chronic comorbid conditions were identified by ICD-8 (1977-93) and ICD-10 (1994-) codes and included all those listed in the Charlson comorbidity indices. ${ }^{14}$ Previous major osteoporotic fractures (that is, fractures of the spine, forearm, humerus, hip, pelvis, and lower leg) were also ascertained with ICD-10 codes.

Baseline characteristics for the longitudinal cohort were those present at the time of the first alendronate prescription (cohort method), while characteristics (that is, confounders) adjusted for in the nested case-control studies were defined at the time of the fracture event to adhere to case-control methods.

The study design was intended to avoid confounding by indication through inclusion of patients who had been prescribed only alendronate, a drug that is exclusively used for osteoporosis and for which Danish reimbursement criteria require patients to have low bone mineral density or have experienced low trauma fractures. Matching procedures were used to ensure that nested fracture cases and controls were of the same age and sex and that risk time was balanced. Residual unbalancing in baseline comorbid conditions, history of fracture before treatment, and key drug exposures were examined by including these as covariates in the conditional logistic regression analyses (see statistical methods).

\section{Statistical methods}

We used SAS v 9.4 (SAS Institute, Cary, NC) for matching for the two nested case-control studies using the gmatch macro (Mayo Clinic, 2003). Fracture cases were individually matched N:1 for year of birth (maximum distance one year), sex, and year of initiation of alendronate treatment to non-cases. Both cases and noncases were drawn from the cohort of alendronate users with no requirement to still be using alendronate as this is handled as an exposure variable in the subsequent logistic regression analysis. We used the TIME variable in the matching routine to ensure controls remained alive at the time that their fracture case experienced their fracture outcome. We were able to use 5:1 matching in the nested case-control study of the rarer outcome (subtrochanteric and shaft fractures) and 3:1 matching for the hip fracture analysis. Case-control analyses were done with conditional logistic regression analysis (SPSS v 19.0) with results shown as crude and adjusted odds ratios with $95 \%$ confidence intervals. We prespecified medication possession ratio and dose year cut-off points based on previous analyses of observational data, ${ }^{15} 16$ where medication possession ratio $<80 \%$ (and $<50 \%$ ) with alendronic acid have been associated with a reduced anti-fracture efficacy.

We planned sensitivity analyses in which we included subsequent use of osteoporosis drugs other than alendronate, and subanalyses examining subtrochanteric and shaft fractures separately rather than combined. For the former, we also separately assessed inclusion of drugs that have (other bisphosphonates and denosumab) or have not (parathyroid hormone analogues, raloxifene, strontium ranelate) been linked to atypical fractures in the current literature. The rationale here was that the first class of drugs could add additional risk $^{17}$ whereas the second class of drugs would either be neutral or, in the case of teriparatide, potentially reduce risk or promote healing. ${ }^{18}$

There were no post hoc or unplanned subgroup analyses. We compared baseline descriptive characteristics with $t$ tests and $\chi^{2}$ tests as appropriate, using a critical significance level of $5 \%$ and two sided tests throughout.

We used the risk reduction for hip fractures associated with medication possession ratio $>80 \%$ to estimate the number of hip fractures prevented in the harm:benefit calculations as numbers cannot be directly observed, and we preferred to use real world data rather than use the larger relative risk reduction found in the phase III trials. Number needed to treat and number needed to treat to harm were calculated from the observed event rates in separate time windows of the alendronate adherent cohort analysis, with 0-5 years of adherence as the comparator,and with the case-control adjusted odds ratios as an approximation for relative risk reduction (where odds ratios $<1$ ) or increase (where odds ratios $>1$ ) as a function of duration of treatment. ${ }^{19}$ Number needed to treat to harm is the same metric based on excess adverse events for those analyses where the adjusted odds ratios was $>1$, regardless of significance, to provide a "worst case scenario" (none of the obtained odds ratios were $>1$ and significant). No power calculation was performed as we included all eligible patient records.

\section{Patient involvement}

No patients were involved in setting the research question or the outcome measures, nor were they involved in developing plans for recruitment, design or implementation of the study. No patients were asked to advise on interpretation or writing up of results. Patient charities will be involved in the dissemination of our findings to relevant audiences in the UK (via the National Osteoporosis Society) and Denmark (via the Danish Osteoporosis Society).

\section{Results}

The 61990 alendronate users who made up the study cohort were followed for fracture outcomes for a median follow-up time of 6.9 years (range 0-17.9, interquartile range 4.1-9.0), making a total of 418430 person years. During this time $1428 / 61990$ (2.3\%; incidence rate $3.4 / 1000$ person years, $95 \%$ confidence interval 3.2 to 3.6) sustained a subtrochanteric and femoral shaft fracture, and 6784/61990 (10.9\%; 16.2/1000 person years, 15.8 to 16.6) sustained a hip fracture. These constituted the cases for the two nested case-control analyses (fig 1). The mean age at subtrochanteric and femoral shaft fracture was 75 (SD 9.5) and at hip fracture was 76.5 (SD 8.9). The cumulative incidence of the two types of fracture is shown in fig 2. Table 1 shows the baseline characteristics for the study cohort 


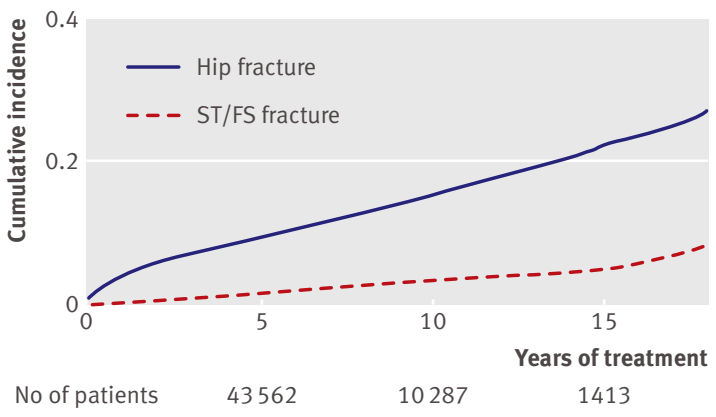

Fig 2 | Kaplan-Meier cumulative incidence plot of hip fracture and subtrochanteric and femoral shaft fracture (ST/FS) as function of time for all people treated with alendronate irrespective of adherence

\begin{tabular}{|c|c|}
\hline & $\begin{array}{l}\text { Full alendronate } \\
\text { cohort }(n=61990)\end{array}$ \\
\hline Mean (SD) age (years) & $72.1(10.0)$ \\
\hline Women & $51558(83.2)$ \\
\hline \multicolumn{2}{|l|}{ Charlson index: } \\
\hline 0 & $30150(48.6)$ \\
\hline 1 & $4247(6.9)$ \\
\hline 2 & $13953(22.5)$ \\
\hline$\geq 3$ & $13640(22.0)$ \\
\hline \multicolumn{2}{|l|}{ Main comorbid conditions: } \\
\hline \multicolumn{2}{|l|}{ Any fracture } \\
\hline Major osteoporotic fracture & $19261(31.1)$ \\
\hline \multicolumn{2}{|c|}{ Fracture of pelvis, femur, or lower leg } \\
\hline Diabetes & $3085(5.0)$ \\
\hline Chronic kidney disease & $449(0.7)$ \\
\hline Chronic pulmonary disease & $11942(19.3)$ \\
\hline Previous myocardial infarction & $3998(6.4)$ \\
\hline \multicolumn{2}{|l|}{ Drug history: } \\
\hline Prednisolone in past year & $15377(24.8)$ \\
\hline
\end{tabular}

(at start of treatment). Table 2 shows clinical characteristics for both matched nested case-control studies.

\section{Cohort study of long term rates of fracture in} adherent alendronate users

Out of the 61990 alendronate users who made up the cohort, 18242 (29.4\%) completed five years of treatment, with a medication possession ratio of $80 \%$ or more, 2465 (4.0\%) completed 10 years, and fewer than 1000 patients completed 14 years or more. For the first 10 years, rates of hip fracture declined from an initial $36.2 / 1000$ person years in the first year of treatment to a stable 10-15 per 1000 patient years (fig 3), while the total rate of subtrochanteric and femoral shaft fractures remained stable at 2.7-4.6/1000 patient years.

\section{Case control study of subtrochanteric and shaft fractures}

Table 2 shows the characteristics of the 1428 patients who experienced a subtrochanteric and femoral shaft fracture and their 6825 age and sex matched (5:1) cohort controls (alendronate users who did not experience the outcome of interest). Briefly, those with subtrochanteric and femoral shaft fracture had a higher Charlson comorbidity index, were more likely to have a history of major osteoporotic fractures, and have diabetes, chronic pulmonary disease, and previous myocardial infarction. The frequency of use of proton pump inhibitors was also higher among cases.

Conditional logistic regression analysis (table 3 ) showed a reduced risk of subtrochanteric and femoral shaft fracture in highly adherent users (medication possession ratio $>80 \%$ ) compared with poor adherers $(<50 \%$, reference category; odds ratio 0.88 , $95 \%$ confidence interval 0.77 to $0.99 ; \mathrm{P}=0.05)$. This changed, however, when we adjusted for comorbid conditions (adjusted odds ratios 0.90, 0.78 to 1.03;

Table 2 | Comorbid conditions and comedications at time of event in two nested case-control studies. Differences in comorbid conditions and comedications between outcome groups, combined with data on degree of alendronate exposure, drive conditional logistic regression analyses in subsequent tables. Figures are numbers (percentage) of patients unless specified otherwise

\begin{tabular}{|c|c|c|c|c|c|c|}
\hline & \multicolumn{3}{|c|}{ Subtrochanteric and femoral shaft fracture } & \multicolumn{3}{|l|}{ Hip fracture } \\
\hline & Cases $(n=1428)$ & Controls $(\mathrm{n}=6825)$ & Pvalue & Cases $(n=6784)$ & Controls $(n=19952)$ & Pvalue \\
\hline Mean (SD) age (years) & $79.6(9.4)$ & $79.7(9.4)$ & $N A^{*}$ & $80.2(8.9)$ & $80.1(8.8)$ & $N A^{\star}$ \\
\hline Women & $1258(88.1)$ & $6056(88.7)$ & $N A^{*}$ & $5980(88.1)$ & $17605(88.1)$ & $N A^{*}$ \\
\hline \multicolumn{7}{|l|}{ Charlson index: } \\
\hline 0 & $409(28.6)$ & $2530(37.1)$ & \multirow{4}{*}{$<0.001$} & $2092(30.8)$ & 7504 (37.6) & \multirow{4}{*}{$<0.001$} \\
\hline 1 & $102(7.1)$ & $442(6.5)$ & & $514(7.6)$ & $1475(7.4)$ & \\
\hline 2 & $323(22.6)$ & $1593(23.3)$ & & $1485(21.9)$ & $4573(22.9)$ & \\
\hline$\geq 3$ & $594(41.6)$ & $2260(33.1)$ & & $2693(39.7)$ & $6400(32.1)$ & \\
\hline \multicolumn{7}{|l|}{ Main comorbid conditions: } \\
\hline Any fracture & $1091(76.4)$ & $3447(50.5)$ & $<0.001$ & $4287(63.4)$ & $9061(45.9)$ & $<0.001$ \\
\hline Major osteoporotic fracture & $987(69.1)$ & $2967(43.5)$ & $<0.001$ & $3778(55.8)$ & 7709 (39.1) & $<0.001$ \\
\hline Fracture of pelvis, femur, or lower leg & $364(25.5)$ & $1034(15.2)$ & $<0.001$ & $1260(18.6)$ & $2547(12.9)$ & $<0.001$ \\
\hline Diabetes & $125(8.8)$ & $400(5.9)$ & $<0.001$ & $496(7.3)$ & $1204(6.1)$ & $<0.001$ \\
\hline Chronic kidney disease & $25(1.8)$ & $74(1.1)$ & 0.04 & $125(1.8)$ & $248(1.3)$ & $<0001$ \\
\hline Chronic pulmonary disease & $325(22.8)$ & $1382(20.2)$ & 0.03 & $1497(22.1)$ & $4001(20.3)$ & 0.001 \\
\hline Previous myocardial infarction & $144(10.1)$ & $541(7.9)$ & 0.008 & $648(9.6)$ & $1726(8.7)$ & 0.04 \\
\hline \multicolumn{7}{|l|}{ Drug history (in past year): } \\
\hline Prednisolone & $252(17.6)$ & $1336(19.6)$ & 0.10 & $1255(18.5)$ & $4146(20.8)$ & $<0.001$ \\
\hline Proton pump inhibitors & $483(33.8)$ & $1948(28.5)$ & $<0.001$ & $2226(32.8)$ & $5488(27.5)$ & $<0.001$ \\
\hline Thiazides & $350(24.5)$ & $1751(25.7)$ & 0.38 & $1762(26.0)$ & $5187(26.0)$ & 0.97 \\
\hline
\end{tabular}

\footnotetext{
*Age and sex matched analysis, P values not calculated.
} 


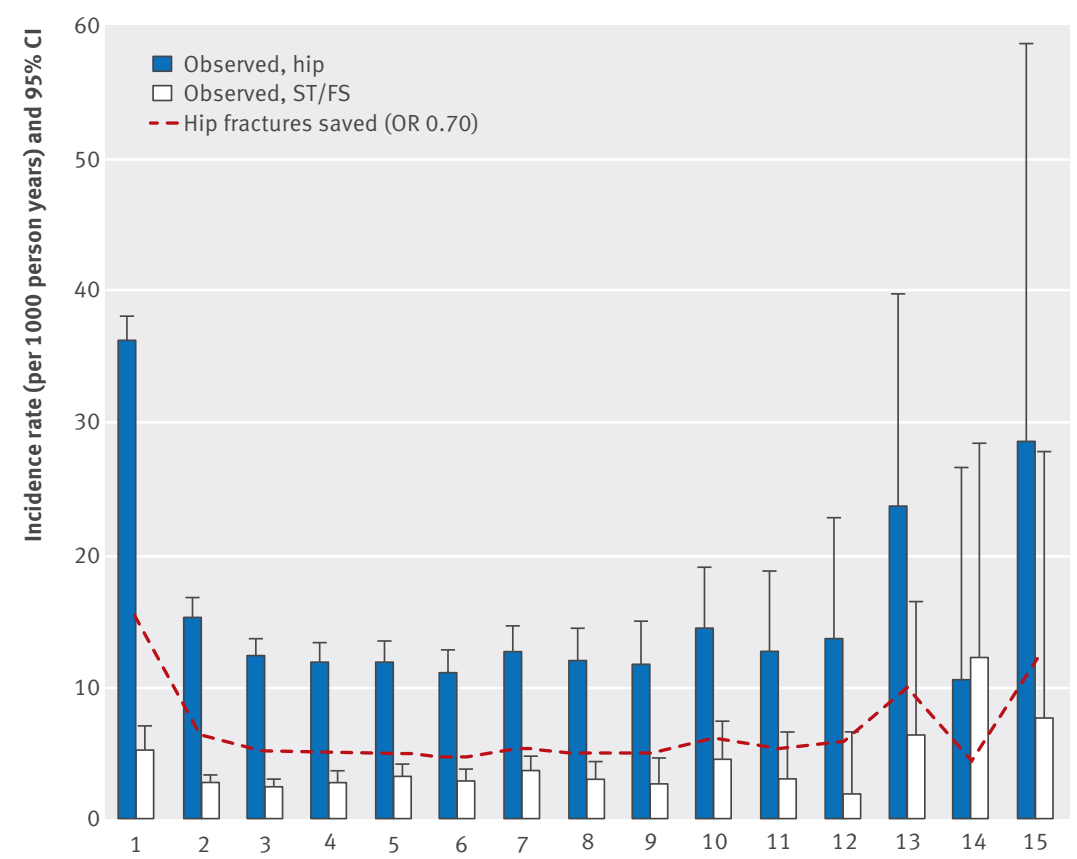

Year of treatment (MPR $280 \%$ )

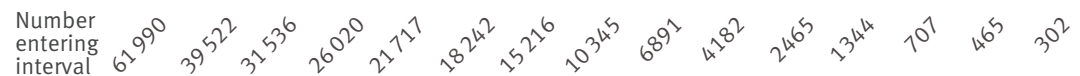

Fig 3 | Fracture rates and 95\% confidence intervals per 1000 patient years for hip fractures and subtrochanteric and femoral shaft fractures in highly adherent alendronate users (medication possession ratio $>\mathbf{8 0} \%$ ). Hip fractures prevented calculated based on OR of 0.70 as derived from nested case-control analyses (see text for details)

$\mathrm{P}=0.11)$. We did not find any association with cumulative use of alendronate (adjusted 0.72, 0.45 to 1.14; $\mathrm{P}=0.16$, for users of $\geq 10$ dose years) or with current use compared with past use (0.92, 0.79 to $1.07 ; \mathrm{P}=0.27)$. Sensitivity analyses in which subsequent use of other osteoporosis drugs including potent antiresorptives such as denosumab did not modify these findings. The risk of subtrochanteric and femoral shaft fractures was significantly higher in patients with comorbid conditions, including diabetes, and in patients who filled prescriptions for proton pump inhibitors. Use of more than 10 dose years of alendronate was associated with a decreased risk (0.43, 0.22 to 0.83 ; $\mathrm{P}=0.01$; fig 4) for subtrochanteric femur fractures but no reduction in the risk of shaft fractures $(1.16,0.60$ to 2.23; $\mathrm{P}=0.66)$.

\section{Nested case-control study of hip fractures}

Conditional logistic regression (table 4) in the nested case control study of the 6784 users who experienced a hip fracture in the follow-up period, matched by age and sex to 19952 users who did not experience hip fractures, showed a reduced risk of hip fracture in current compared with past users (adjusted odds ratios 0.70, 95\% confidence interval 0.65 to $0.77 ; \mathrm{P}<0.001$ ). Optimal compliance (users with medication possession ratio $>80 \%$; 0.73, 0.69 to $0.79 ; \mathrm{P}<0.001)$ as well as cumulative use for $5-10$ dose years $(0.74,0.67$ to 0.83 ; $\mathrm{P}<0.001)$ or $>10$ dose years $(0.74,0.55$ to $0.97 ; \mathrm{P}=0.03$ ) were associated with a reduced risk of hip fracture. Sensitivity analyses that excluded switchers to other osteoporosis drugs did not alter the findings. The risk was higher if patients had previous major osteoporotic fractures, used proton pump inhibitors, or had comorbid conditions such as diabetes or kidney disease.

\section{Long term harm:benefit model}

In each year, the upper 95\% confidence limit for subtrochanteric and femoral shaft fractures was lower than the observed rate of hip fractures in up to 13 years of alendronate adherence (fig 3). Table 5 summarises the fracture rates during 0-5 years, 5-10 years, and $\geq 10$ years of alendronate adherence. Combination of these observed event rates of subtrochanteric and femoral shaft and hip fractures and the odds ratios adjusted for confounding from the nested case control studies yielded a number needed to treat for hip fractures of 38 people treated for an additional five years to prevent one hip fracture after both $\geq 5$ and $\geq 10$ years of alendronate adherence, compared with people with less than five years of adherence. For subtrochanteric and femoral shaft fractures, we found a number needed to treat to harm of 1449 people treated for an additional five years to account for one additional subtrochanteric and femoral shaft fracture in the 5-10 year scenario. The $\geq 10$ year scenario yielded a risk reduction compared with less than five years of alendronate adherence and a number needed to treat of 193 people treated for an additional five years to avoid one subtrochanteric and femoral shaft fracture. Both time scenarios indicated an overall lower fracture burden at the hip and femur with long alendronate adherence compared with less than five years of adherence.

\section{Discussion}

\section{Principal findings}

This study provides real world data on the incidence rates of subtrochanteric and diaphyseal femur fractures with verified long term adherence to alendronate, a subset of femur fractures that captures the total rate of atypical and typical femur fractures to be offset against hip fractures prevented. In addition, two nested case control studies explored risk factors for subtrochanteric and femoral shaft and hip fractures, respectively, and showed that while use of alendronate in excess of 10 dose years was associated with a $30 \%$ lower risk of hip fracture, there was no increase in the risk of fractures of the subtrochanteric femur or femoral shaft. It is important to appreciate that the two case-control studies incorporate an adjustment for confounders such as previous fractures, diabetes, and proton pump inhibitors that are not considered in the cohort analysis. Hence the purpose of the cohort analysis is to provide absolute fracture rates for harm:benefit modelling, whereas the nested case-control studies are optimal for estimating the influence of adherence (medication possession ratio) and cumulative use (dose years) on the risk of fractures when we take into account other patient factors that might be unbalanced between fracture cases and matched non-fracture controls. 


\begin{abstract}
Table 3 | Nested case-control analysis of exposure to drug treatment and comorbid conditions as predictor of subtrochanteric or shaft fractures of femur in treatment naive adults initially starting alendronate in Denmark. Table summarises several multivariate conditional logistic regression analyses of exposure in terms of current user status, adherence, or number of dose years
\end{abstract}

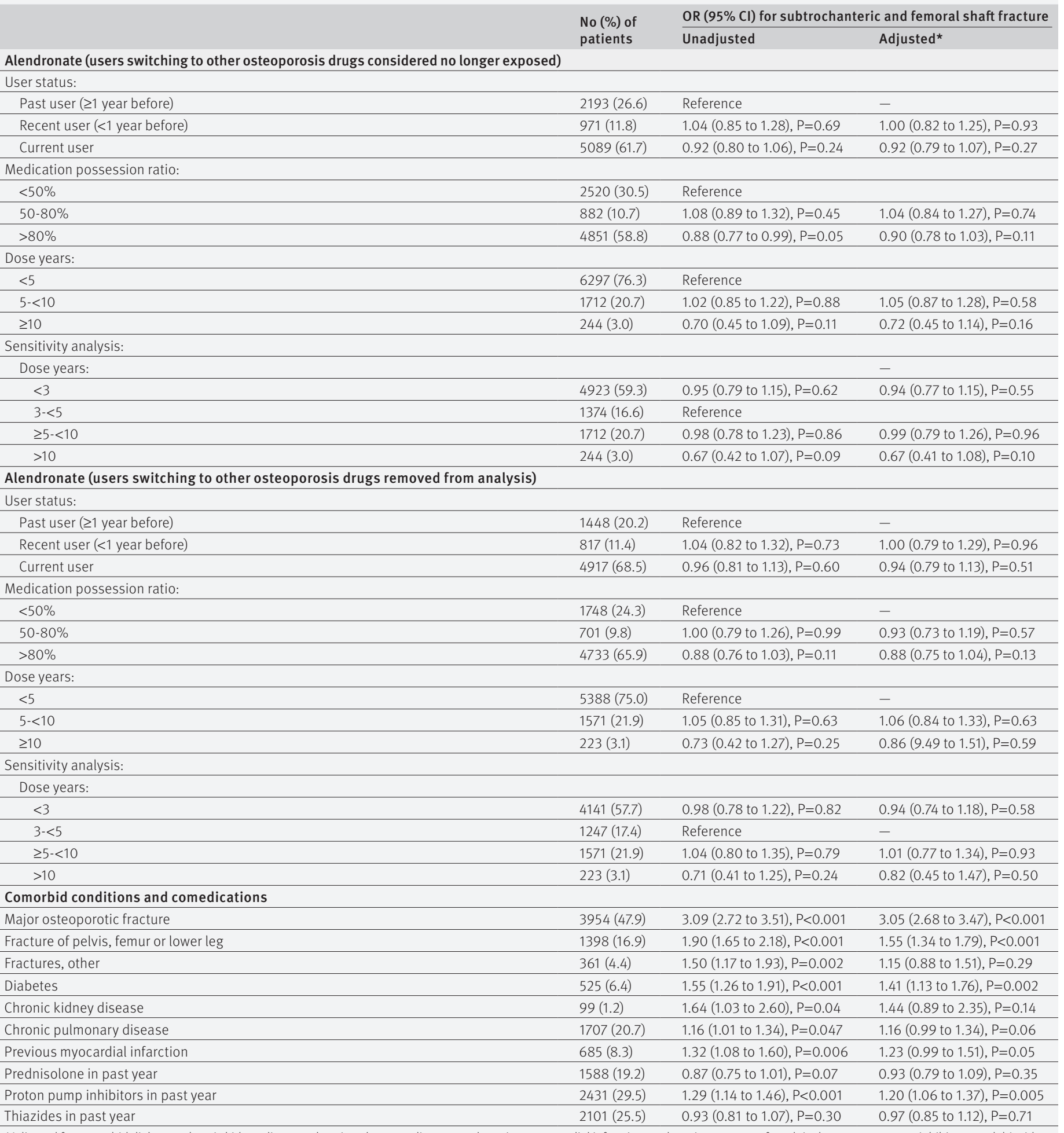

${ }^{*}$ Adjusted for comorbid diabetes, chronic kidney disease, chronic pulmonary disease, and previous myocardial infarction, and use in past year of prednisolone, proton pump inhibitors, and thiazides.

From a pharmacoepidemiological point of view we then proceeded to assume a worst case scenario in which all subtrochanteric and femoral shaft fractures observed were considered atypical and attributable to bisphosphonate treatment-assuming a rate of zero in the background population-and applying the upper 95\% confidence limit in the harm:benefit models rather than the observed rates. With this extreme scenario there was still a net saving in fractures at the hip and femur for up to 10 years of treatment with high adherence. 


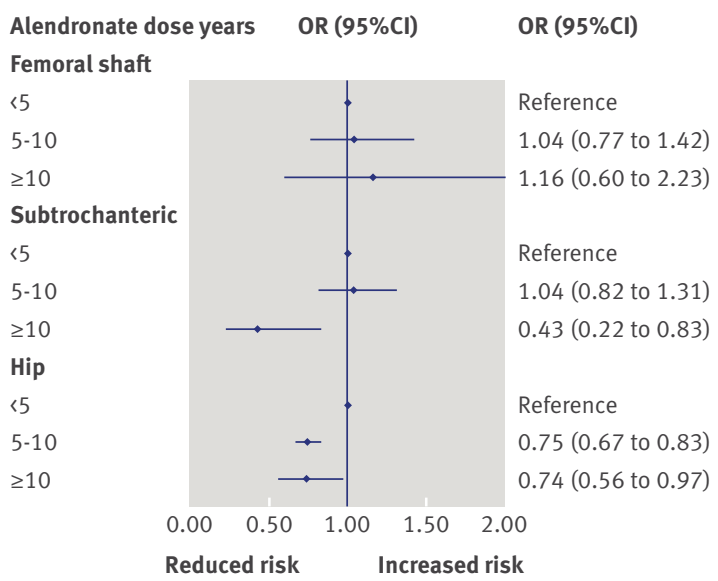

Fig 4 | Subanalysis of femoral shaft fractures, subtrochanteric fractures, and hip fractures. Nested case-control analysis adjusted for covariates in tables 2 and 3

Comparison with other/previous studies

Atypical femur fractures are thought to be linked to impaired properties of bone material with hypermineralisation, loss of flexibility, increased hardness, and loss of toughness. It is unknown if there is a genetic susceptibility to such fractures, but the risk is clearly higher in people of South East Asian origin, ${ }^{20}$ and several groups have found links to femur shape and hip geometry. ${ }^{21-23}$ It is not clear if every patient treated for osteoporosis could develop atypical femur fractures or if only a small minority of patients could be at risk so identification of strong clinical risk factors could be helpful. Previous studies have shown that the use of proton pump inhibitors and diabetes mellitus are associated with subtrochanteric and shaft fracture, ${ }^{24-26}$ and we confirmed that they were strong risk factors in our study. A reduced risk of fracture in prednisolone users was seen only in the unadjusted analyses but as it was attenuated in the multivariable models this observation was probably caused by confounding.

Recent reports from Sweden indicate that the relative risk of atypical femur fractures is increased 126-fold (11 per 10000 patient years) after as little as four years of exposure to bisphosphonates. ${ }^{10}$ The rate of classic hip fractures was almost identical among bisphosphonate users in the Swedish analysis and in our study, at about 1500 per 10000 person years. We observed only 46 subtrochanteric and femoral shaft fractures per 10000 person years in the 10th year of alendronate treatment, but no risk increase with time or cumulative dose whether or not we adjusted for comorbid conditions and comedications. Because of the extremely low rate of atypical femur fractures in the general population ( 0.09 per 10000 reported for Sweden) the risk of atypical femur fractures could increase by more than 100 -fold in four years of bisphosphonate use with no impact on the burden of femoral and hip fracture. The rate of hip fractures of 1500 per 10000 per year in Danish and Swedish bisphosphonate users is almost 1700 times higher than the rate of atypical femur fractures in non-bisphosphonate users reported from Sweden.
Our study provides reassurance that the total risk of subtrochanteric and femoral shaft fractures does not increase over the first 10 years of high adherence to alendronate in Danish adults with osteoporosis, implying that increases in atypical femur fractures are offset by decreases of at least the same size in the risk of non-atypical subtrochanteric and femoral shaft fractures. Atypical femur fractures are not undermining the benefits on hip fracture; they are not even discernible as an increase in subtrochanteric and femoral shaft fractures. Though we observed an increase in the rates of both subtrochanteric and femoral shaft fractures and hip fractures after 13 years or more, the case-control analysis suggests that this might not be indicative of a change in treatment effect but of a more adverse risk factor profile in those with long term adherent use.

\section{Strengths and limitations}

The key limitation of the present study is that radiographs were not available so the absolute harm rates reported here are inflated by inclusion of non-atypical subtrochanteric fractures and shaft fractures. The proportion of subtrochanteric and femoral shaft fractures among bisphosphonate users accounted for by atypical femur fractures varies somewhat between studies. The updated report from Sweden ${ }^{10}$ identified 172 atypical femur fractures among 5475 subtrochanteric and femoral shaft fractures (3.1\%), though 4218 fractures were classed as irrelevant on radiological review, either because of prostheses or a condylar or trochanteric location, despite the ICD-10 coding applied, bringing the number of atypical femur fractures among true subtrochanteric and femoral shaft fractures in a mechanically unaltered femur to $13.4 \%$. The Kaiser California study ${ }^{27}$ identified 142 atypical femur fractures (3.5\%) among 4036 subtrochanteric and femoral shaft fractures. By contrast, the Kaiser Northwest study reported that $38 \%$ of femoral shaft fractures and $8.6 \%$ of reviewed femur radiographs fulfilled the criteria for classification as atypical femur fractures. ${ }^{28}$

Though use of drug adherence and cumulative dose as the driver for the analysis is a classic method in pharmacoepidemiology, it is important to recognise the limitations. Patients who adhere to treatment might differ on unmeasured confounders-such as education, nutrition, or exercise habits-from those who do not adhere to treatment. This would lead to residual confounding, which could bias our findings; such issues, however, are present in all observational studies and can be resolved only in long term randomised controlled trials. Also, given current recommendations about the duration of treatment with bisphosphonates, patients who receive treatment for the longest might do so because they have strong risk factors for fracture, and this could lead to a higher risk of fracture after 5-10 years of treatment. The opposite might also be true, as patients who adhere to treatment might be risk aware and follow other recommendations (that is, "healthy adherers") commonly given together with the prescription of 
Table 4 | Nested case-control analysis of exposure to drug treatment and comorbid conditions as predictor of hip fractures in treatment naive adults initially starting alendronate in Denmark. Table summarises several multivariate conditional logistic regression analyses examining exposure in terms of current user status, adherence, or number of dose years

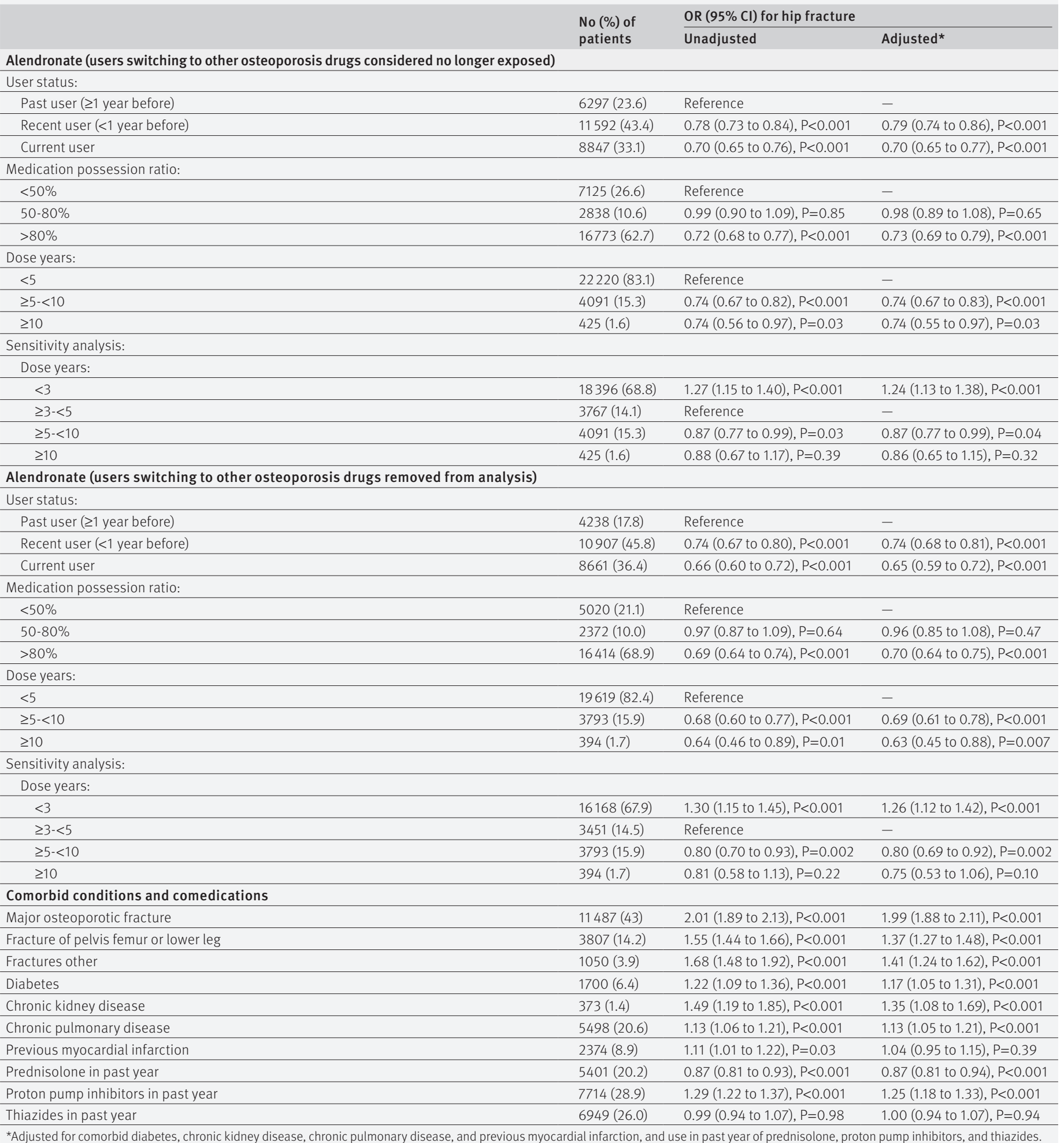

anti-osteoporosis drugs (for example, physical activity, lifestyle modification, dietary calcium intake, etc). Excess mortality in those with the lowest bone mineral density and highest fracture risk might contribute as well. It is important to be aware that unlike the case-control analyses, the rates shown in fig 3 are not adjusted or standardised for age, sex, or comorbidity. An additional limitation of our data is the lack of information on use of calcium/vitamin D supplements, as these are almost exclusively purchased over the counter in Denmark, making it impossible for us to study them from prescription data. 


\begin{tabular}{|c|c|c|c|}
\hline & \multicolumn{3}{|c|}{ Treatment duration (years) } \\
\hline \multicolumn{4}{|c|}{ Subtrochanteric and femoral shaft fracture } \\
\hline No of patients & 566 & 157 & 19 \\
\hline Case-control odds ratios (table 3 ) & Reference & 1.04 & 0.70 \\
\hline Number needed to treat $\dagger$ & Reference & - & 193 \\
\hline Number needed to harmt & Reference & 1449 & - \\
\hline \multicolumn{4}{|l|}{ Hip fracture } \\
\hline No of patients & 3289 & 562 & 66 \\
\hline Number needed to treat $\dagger$ & Reference & 38 & 38 \\
\hline Number needed to harmt & Reference & - & - \\
\hline \multicolumn{4}{|c|}{$\begin{array}{l}\text { *Incidence rate for comparison based on observed absolute event rates. Lower incidence rate does not necessarily imply lower odds ratios as patients } \\
\text { with different duration of use might differ on risk characteristics. } \\
\text { tNumber needed to treat and number needed to harm express number of patients who must be treated for another additional five years to prevent (number } \\
\text { needed to treat) or add (number needed to harm) one additional fracture respectively. Comparator is treatment for } 0-5 \text { years. Case-control odds ratio } \\
\text { that contribute to calculation of these is age and sex matched metric adjusted for comorbid conditions and comedications (see tables } 2 \text { and } 3 \text { for details). }\end{array}$} \\
\hline
\end{tabular}

Our study population was almost exclusively North European, and the results might not apply to other ethnic groups, particularly to patients of South Asian heritage who might be at particular risk of atypical femur fractures. ${ }^{20}$ Also, we focused on subtrochanteric/shaft femoral fractures but did not study the potential increase in risk of another relevant potential bone undesired effect: osteonecrosis of the jaw. Finally, though we provide quantitative data on the benefits and risks of treatment in terms of burden of femur fracture, we did not account for the high morbidity associated with atypical fractures, which can be high and even exceed that of hip fracture. Recent research, however, has shown that the mortality associated with atypical femoral fractures is lower than previously assumed ${ }^{29}$ and clearly lower than that after a hip fracture. More data are therefore needed for an accurate evaluation of the impact of such fractures on patients' morbidity and mortality. ${ }^{30}$

The present type of study is more informative for deriving benefit and harm rates than for disentangling the mechanisms underlying atypical femur fractures. The most important strength of the study is the quality and duration of drug exposure data, which allows researchers to work with almost two decades of drug prescription data, encompassing the early years of alendronate becoming established as a therapeutic option in Denmark. We used an elaborate user only study design to eliminate drug channelling bias and embedded two case control studies in a longitudinal open cohort study of alendronate use to achieve optimum statistical power for this rare fracture outcome. By using nested case-control studies we sought to overcome the colinearity challenge that would have been posed in a cohort study with Cox proportional hazards models incorporating a time dependent covariate because there would be strong colinearity of time and cumulative dose. Moreover, if risks change abruptly with duration of use then the proportional hazards assumption would not hold. The nested case-control setup allowed us to enter strength of alendronate exposure in several conventional ways used in pharmacoepidemiology (refill compliance, dose years, and present versus past use) to identify a risk association if one was indeed present. The analysis did not only recapture those risk factors already known to predict fractures in other studies, it also showed risk reductions for hip fractures consistent with those observed in other real world settings. ${ }^{15} 31$

Regarding the potential benefits of treatment with bisphosphonates, our data are consistent with findings of previous systematic reviews of randomised controlled trials: a Cochrane review published in 2008 reported on a $40 \%$ relative risk reduction of hip fracture after up to four years of treatment with alendronic acid. ${ }^{32}$ This protective effect was stronger (over 50\% risk reduction) in secondary fracture prevention. In our study, in which almost one in three patients received secondary prevention, we observed an overall almost $30 \%$ reduction on risk of hip fracture associated with good compliance. These protective effects extended for up to in excess of 10 years, complementing previous randomised studies included in the Cochranesystematic review ${ }^{32}$ with long term (observational) effectiveness data.

There is an important difference between the present case-control analyses and those that have been conducted in the context of most radiology review studies of atypical femur fractures. Our analyses used a classic case-control design in which patients with an outcome were compared with similar patients (for instance, users of the same drug of same age, sex, and who started treatment in the same year) without this outcome to identify factors associated with the risk of developing the outcome in question. Previous casecontrol studies have relied on patients with other 
femoral fractures as controls for patients with atypical femur fractures. $.^{24} 283-35$ This approach produces an odds ratio that measures the likelihood that patients who sustain fractures of the femur do so in the form of atypical fractures, with an external validity that extends to patients with hip and femur fracture alone and not to bisphosphonate users in general. This is akin to identifying risk factors for breast cancer solely from a case comparison with women with colon cancer.

\section{Conclusions and policy implications}

In summary, the present nationwide register based cohort and case-control studies with long term adherence data found that use of alendronate in excess of 10 dose years was associated with a confounder adjusted $30 \%$ lower risk of hip fracture and no increase in the risk of fractures of the subtrochanteric femur and femoral shaft. In addition, we have shown that even in the worst case scenario (assuming 100\% of subtrochanteric and femoral shaft fractures are atypical and secondary to bisphosphonate use and making no allowance for the higher prevalence of comorbid conditions in these patients) the number of atypical femur fractures remains too low to offset the benefits on hip fracture in patients with long term alendronate use up to 10 years. Furthermore, realistic comorbidity adjusted number needed to treat to harm and number needed to treat scenarios suggested that use of alendronate for more than 10 years remains favourable at the femur and hip. Long term exposure data linked to radiologically adjudicated fracture outcomes are recommended to accurately determine the true, and possibly even lower, rate of atypical femur fractures.

\section{AUTHOR AFFILIATIONS}

${ }^{1}$ Odense Patient Data Explorative Network, Department of Clinical Research, University of Southern Denmark, J.B. Winsløws Vej 9 A, 3. Sal, DK-5000, Odense, Denmark

2Department of Medicine, Holbæk Hospital, Smedelundsgade 60, 4300 Holbæk, Denmark

${ }^{3}$ Department of Cardiology, Nephrology and Endocrinology, Hillerød Hospital, Dyrehavevej 29, 3400 Hillerød, Denmark, Pia Eiken

4Faculty of Health and Medical Sciences, University of Copenhagen, Blegdamsvej 3B, 2200 Copenhagen, Denmark, Pia Eiken

${ }^{5}$ Oxford NIHR Musculoskeletal Biomedical Research Unit, Nuffield Department of Orthopaedics, Rheumatology, and Musculoskeltal Sciences (NDORMS), University of Oxford, Botnar Research Centre, Oxford OX3 7LD, UK

${ }^{6}$ Musculoskeletal Research Unit, IMIM-Parc de Salut Mar and RETICEF, Universitat Autònoma de Barcelona and Instituto Carlos III (FEDER Research Funds), Passeig Marítim 25-29, 08003 Barcelona, Spain

${ }^{7}$ Academic Unit of Bone Metabolism (AUBM), Northern General Hospital and University of Sheffield, Sheffield S5 7AU, UK

Contributors: All authors contributed to the design of the study, the interpretation of the results, and reviewed the manuscript. BA performed the statistical analysis and is guarantor. BA and DP-A wrote the first draft of the manuscript. RE and DP-A are joint senior authors. Funding: This research received no specific grant from any funding agency in the public, commercial, or not-for-profit sectors.

Competing interests: All authors have completed the ICMJE uniform disclosure form at www.icmje.org/coi_disclosure.pdf and declare: no support from any organisation for the submitted work; BA receives institutional research grants and has contracts with Novartis and UCB, past institutional research contracts with Amgen and NPS
Pharmaceuticals, and past payment for membership of advisory boards from Nycomed, Merck, and Amgen; PE reports grant support from Eli Lilly and payment for educational presentations for Amgen and Eli Lilly, pro bono educational presentations for Boehringer Ingelheim, payment for membership of advisory boards from Amgen, Eli Lilly, and Merck, and stock ownership in Novo Nordisk; DP-A reports institutional research grants from Amgen and Servier and support for conference attendance and speaker fees paid to his institution; RE reports institutional research grants and personal fees from Amgen, IDS, Alexian, and Roche, institutional research grants from Astra Zeneca, and speaker or consulting fees from Bayer, Fonterra, Janssen, Eli Lilly, Ono Pharma, Alere, Teijin Pharm, D-STAR, and GSK nutrition.

Ethical approval: The study was approved by Statistics Denmark (project 702538) and by the Medicines Agency branch of the Danish National Board of Health. Analyses were conducted via VPN exclusively on de-identified microdata hosted with Statistics Denmark with no access to patients' names, social security numbers, or other identifiers.

Transparency: The lead authors affirm that the manuscript is an honest, accurate, and transparent account of the study being reported; that no important aspects of the study have been omitted; and that any discrepancies from the study as planned (and, if relevant, registered) have been explained.

\section{Data sharing: No additional data available.}

This is an Open Access article distributed in accordance with the Creative Commons Attribution Non Commercial (CC BY-NC 3.0) license, which permits others to distribute, remix, adapt, build upon this work non-commercially, and license their derivative works on different terms, provided the original work is properly cited and the use is non-commercial. See: http://creativecommons.org/licenses/ by-nc/3.0/.

Russell RG. Bisphosphonates: the first 40 years. Bone 2011;49:219. doi:10.1016/j.bone.2011.04.022.

2 Shane E, Burr D, Abrahamsen B, et al. Atypical subtrochanteric and diaphyseal femoral fractures: second report of a task force of the American Society for Bone and Mineral Research. J Bone Miner Res 2014:29:1-23. doi:10.1002/jbmr.1998.

3 Järvinen TL, Michaëlsson K, Aspenberg P, Sievänen H. Osteoporosis: the emperor has no clothes. J Intern Med 2015;277:662-73. doi:10.1111/joim.12366.

4 Jha S, Wang Z, Laucis N, Bhattacharyya T. Trends in Media Reports, Oral Bisphosphonate Prescriptions, and Hip Fractures 1996-2012: An Ecological Analysis. J Bone Miner Res 2015;30:2179-87. doi:10.1002/ jbmr.2565.

5 Svedbom A, Hernlund E, Ivergård M, et al. EU Review Panel of IOF Osteoporosis in the European Union: a compendium of countryspecific reports. Arch Osteoporos 2013;8:137. doi:10.1007/ s11657-013-0137-0.

6 Järvinen TL, Michaëlsson K, Jokihaara J, et al. Overdiagnosis of bone fragility in the quest to prevent hip fracture. BMJ 2015;350:h2088. doi:10.1136/bmj.h2088.

7 Black DM, Schwartz AV, Ensrud KE, et al. FLEX Research Group. Effects of continuing or stopping alendronate after 5 years of treatment: the Fracture Intervention Trial Long-term Extension (FLEX): a randomized trial. JAMA 2006;296:2927-38. doi:10.1001/jama.296.24.2927.

8 Black DM, Reid IR, Cauley JA, et al. The effect of 6 versus 9 years of zoledronic acid treatment in osteoporosis: a randomized second extension to the HORIZON-Pivotal Fracture Trial (PFT). J Bone Miner Res 2015;30:934-44. doi:10.1002/jbmr.2442.

9 Reyes C, Hitz M, Prieto-Alhambra D, Abrahamsen B. Risks and Benefits of Bisphosphonate Therapies. J Cell Biochem 2016;117:20-8. doi:10.1002/jcb.25266

10 Schilcher J, Koeppen V, Aspenberg P, Michaëlsson K. Risk of atypical femoral fracture during and after bisphosphonate use. Acta Orthop 2015:86:100-7. doi:10.3109/17453674.2015.1004149.

11 Wang Z, Ward MM, Chan L, Bhattacharyya T. Adherence to oral bisphosphonates and the risk of subtrochanteric and femoral shaft fractures among female medicare beneficiaries. Osteoporos Int 2014;25:2109-16. doi:10.1007/s00198-014-2738-x.

12 Abrahamsen B, Eiken P, Eastell R. Cumulative alendronate dose and the long-term absolute risk of subtrochanteric and diaphyseal femur fractures: a register-based national cohort analysis. / Clin Endocrinol Metab 2010;95:5258-65. doi:10.1210/jc.2010-1571.

13 Johannesdottir SA, Horváth-Puhó E, Ehrenstein V, Schmidt M, Pedersen L, Sørensen HT. Existing data sources for clinical epidemiology: The Danish National Database of Reimbursed Prescriptions. Clin Epidemiol 2012;4:303-13. doi:10.2147/CLEP. S37587.

14 Quan H, Sundararajan V, Halfon P, et al. Coding algorithms for defining comorbidities in ICD-9-CM and ICD-10 administrative data. Med Care 2005;43:1130-9. doi:10.1097/01.mlr.0000182534.19832.83. 
15 Siris ES, Harris ST, Rosen CJ, et al. Adherence to bisphosphonate therapy and fracture rates in osteoporotic women: relationship to vertebral and nonvertebral fractures from 2 US claims databases. Mayo Clin Proc 2006;81:1013-22. doi:10.4065/81.8.1013.

16 Caro JJ, Ishak KJ, Huybrechts KF, Raggio G, Naujoks C. The impact of compliance with osteoporosis therapy on fracture rates in actual practice. Osteoporos Int 2004;15:1003-8. doi:10.1007| s00198-004-1652-z.

17 Selga J, Nuñez JH, Minguell J, Lalanza M, Garrido M. Simultaneous bilateral atypical femoral fracture in a patient receiving denosumab: case report and literature review. Osteoporos Int 2016;27:827-32. doi:10.1007/s00198-015-3355-z.

18 Chiang CY, Zebaze RM, Ghasem-Zadeh A, Iuliano-Burns S, Hardidge A, Seeman E. Teriparatide improves bone quality and healing of atypical femoral fractures associated with bisphosphonate therapy. Bone 2013:52:360-5. doi:10.1016/j.bone.2012.10.006

19 Chatellier G, Zapletal E, Lemaitre D, Menard J, Degoulet P. The number needed to treat: a clinically useful nomogram in its proper context. BMJ 1996;312:426-9. doi:10.1136/bmj.312.7028.426.

20 Lalmohamed A, Vestergaard P, Klop C, et al. Timing of acute myocardial infarction in patients undergoing total hip or knee replacement: a nationwide cohort study. Arch Intern Med 2012;172:1229-35. doi:10.1001/archinternmed.2012.2713.

21 Taormina DP, Marcano Al, Karia R, Egol KA, Tejwani NC. Symptomatic atypical femoral fractures are related to underlying hip geometry. Bone 2014;63:1-6. doi:10.1016/j.bone.2014.02.006.

22 Saita Y, Ishijima M, Mogami A, et al. The fracture sites of atypical femoral fractures are associated with the weight-bearing lower limb alignment. Bone 2014;66:105-10. doi:10.1016/j.bone.2014.06.008.

23 Schilcher J, Howe TS, Png MA, Aspenberg P, Koh JS. Atypical Fracture are Mainly Subtrochanteric in Singapore and Diaphyseal in Sweden: A Cross-Sectional Study. J Bone Miner Res 2015;30:2127-32 doi:10.1002/jbmr.2547.

24 Giusti A, Hamdy NA, Dekkers OM, Ramautar SR, Dijkstra S, Papapoulos SE. Atypical fractures and bisphosphonate therapy: a cohort study of patients with femoral fracture with radiographic adjudication of fracture site and features. Bone 2011;48:966-71 doi:10.1016/j.bone.2010.12.033.
25 Muschitz C, Thaler HW, Dimai HP, et al. Atypical Femoral FracturesOngoing and History of Bone-Specific Therapy, Concomitant Diseases, Medications, and Survival. / Clin Densitom 2015;S1094-6950(15)00126-2.

26 Napoli N, Schwartz AV, Palermo L, et al. Risk factors for subtrochanteric and diaphyseal fractures: the study of osteoporotic fractures. / Clin Endocrinol Metab 2013;98:659-67. doi:10.1210/jc.2012-1896.

27 Dell RM, Adams AL, Greene DF, et al. Incidence of atypical nontraumatic diaphyseal fractures of the femur. J Bone Miner Res 2012:27:2544-50. doi:10.1002/jbmr.1719.

28 Feldstein AC, Black D, Perrin N, et al. Incidence and demography of femur fractures with and without atypical features. J Bone Miner Res 2012;27:977-86. doi:10.1002/jbmr.1550.

29 Kharazmi M, Hallberg P, Schilcher J, Aspenberg P, Michaëlsson K. Mortality After Atypical Femoral Fractures: A Cohort Study. J Bone Miner Res 2016;31:491-7. doi:10.1002/jbmr.2767.

30 Abrahamsen B, Prieto-Alhambra D. Patients With Atypical Femur Fractures Have the Same Mortality as the Background Population-DrugChanneling Bias, Bisphosphonate Effects, and Public Health Implications. J Bone Miner Res 2016;31:488-90. doi:10.1002/jbmr.2801.

31 Gallagher AM, Rietbrock S, Olson M, van Staa TP. Fracture outcomes related to persistence and compliance with oral bisphosphonates. J Bone Miner Res 2008:23:1569-75. doi:10.1359/jbmr.080510.

32 Wells GA, Cranney A, Peterson J, et al. Alendronate for the primary and secondary prevention of osteoporotic fractures in postmenopausal women. Cochrane Database Syst Rev 2008;(1):CD001155.

33 Girgis CM, Sher D, Seibel MJ. Atypical femoral fractures and bisphosphonate use. N Engl J Med 2010;362:1848-9. doi:10.1056/ NEJMc0910389.

34 Schilcher J, Michaëlsson K, Aspenberg P. Bisphosphonate use and atypical fractures of the femoral shaft. N Engl J Med 2011;364:1728-37. doi:10.1056/NEJMoa1010650

35 Meier RP, Perneger TV, Stern R, Rizzoli R, Peter RE. Increasing occurrence of atypical femoral fractures associated with bisphosphonate use. Arch Intern Med 2012;172:930-6. doi:10.1001/ archinternmed.2012.1796.

(c) BMJ Publishing Group Ltd 2016 